Loading

The Journal of the Canadian Game Studies Association

\title{
Le jeu vidéo au Québec
}

\section{The Video Game in Quebec}

\section{Bernard Perron}

Volume 14, Number 23, 2021

Le jeu vidéo au Québec

The Video Game in Quebec

URI: https://id.erudit.org/iderudit/1078725ar

DOI: https://doi.org/10.7202/1078725ar

See table of contents

Publisher(s)

Canadian Game Studies Association

ISSN

1923-2691 (digital)

Explore this journal

Cite this document

Perron, B. (2021). Le jeu vidéo au Québec. Loading, 14(23), 1-14.

https://doi.org/10.7202/1078725ar 


\title{
Le jeu vidéo au Québec The Video Game in Quebec
}

\author{
Bernard Perron \\ Université de Montréal \\ bernard.perron@umontreal.ca
}

\section{Une reconnaissance en courtepointe}

Le Québec demeure certes un haut lieu du jeu vidéo au Canada. Comme le soulignent ou le rapportent les collaborateurs et collaboratrices de ce numéro afin de marquer la reconnaissance et l'intérêt du sujet d'analyse, "la province se distingue sur le marché mondial de l'industrie culturelle la plus lucrative au monde » (Maude Bonenfant et Jonathan Bonneau); elle est " présentement un leader international dans le développement du jeu vidéo dit mainstream, [et] son écosystème de développeurs indie n'est pas en reste » (Jonathan Lessard et Carl Therrien); Montréal est considérée comme la «capitale du jeu vidéo " ${ }^{1}$ (Laureline Chiapello); bref, «[q]uand on pense au jeu vidéo au Québec, c'est immédiatement son industrie qui nous vient à l'esprit » (Adam Lefloïc Lebel).

Or, on connait somme toute peu de choses de l'histoire de cette industrie et du jeu vidéo au Québec. À ma connaissance, outre une réflexion qui prend Montréal pour exemplifier la dynamique de la créativité localisée (Grandadam, Cohendet et Simon, 2012), d'une autre sur le jeu vidéo à Montréal (Pineault, 2015), de courts articles de Jason Della Rocca (2013) et de Jonathan Lessard (2013) dans un numéro sur les jeux indépendants dans Loading... Journal of the Canadian Game Studies Association, ainsi qu'un chapitre sur le Canada de Dominic Arsenault et Louis-Martin Guay dans l'ouvrage Video Games Around the World (Wolf, 2015), la recherche universitaire ${ }^{2}$ sur la production vidéoludique de la Belle Province ne foisonne pas.

Conséquemment, ce numéro veut donner une impulsion aux études sur le jeu vidéo québécois. Il s'inscrit dans la foulée des approches plus locales de l'histoire du 10 art. Melanie Swalwell s'est entre autres faite l'avocate des spécificités culturelles des réalisations vidéoludiques.

Digital game history has usually been told with a focus on the USA or Japan, assuming the uniformity of products and reception worldwide [...]. While early digital games were a global phenomenon, the 1970s and 1980s also saw games produced and consumed in specifically local contexts. Remarkably little is known about the local aspects of digital games history. In contrast to the high profile games targeted by the Digital Game Canon, in local contexts it is not always clear what software existed even as little as 20 years ago; consequently, identifying priorities for preservation becomes a challenge.

Melanie Swalwell, p. 264 (2009) 
On comprend cette nécessité en consultant les quelques ouvrages connus d'histoire générale du jeu vidéo (Herman, [1994] 2009; Kent, 2001; Wolf, 2007 et Donovan, 2010). Seul Leonard Herman mentionne le Québec, et justement pour signaler une spécificité culturelle. En effet, il explique qu'en 1999 le gouvernement de la Belle Province était en colère contre Nintendo et les autres sociétés de jeux vidéo pour une raison particulière, à savoir la Charte de la langue française. "A Quebec charter decreed that any company that did public business in Frenchspeaking Quebec had to sell its products with the French language on the packaging. Any other language, including English, could be included as an option. To alleviate any problems, most companies merely printed their packages with both English and French writing. Videogame companies were the exception » (Herman, 2009, p. 304). Une exception parce que, suivant ce que rapporte l'historien peu au fait du contexte local (et dont le propos demande à être nuancé), ledit produit contenait du texte qu'il aurait tout de même fallu traduire et que les standards techniques de diffusion étant différents entre la France (SECAM) et le Canada (NTSC), il était impossible de simplement importer les jeux français. ${ }^{3}$ Herman conclut en ces termes : « In order to change the companies' policies towards Quebec, the government of Quebec threatened to sue if the policies weren't changed by the end of the year » (p. 304). Mais l'histoire s'arrête là pour lui, qui ne revient pas sur cet événement dans sa rétrospective de l'année 2000 (la dernière année que passe en revue la $3^{\mathrm{e}}$ édition de son livre). Il n'y avait de toute façon rien à dire puisque la menace n'a pas été vraiment mise à exécution; la francisation des jeux vidéo s'est faite lentement au cours de cette décennie, et davantage par l'entremise d'une entente avec le milieu (Asselin, 2009).

Si l'histoire locale est importante pour bien décrire le fait social et le phénomène culturel de masse qu'est le jeu vidéo, on doit s'y atteler sans trop tarder si l'on souhaite profiter d'un atout que possède le $10^{\mathrm{e}}$ art.

When it comes to media histories, video game historians enjoy a rare luxury: they still have access to a vast amount of individuals who are willing to be interviewed, and otherwise assist in the process of documenting the evolution of the medium.

Carl Therrien, n.p. (2015)

Bien que, comme le note Carl Therrien ensuite, le témoignage oral doive être mis en perspective si l'on veut expliquer les événements et construire des récits qui soient justes, il demeure une base extrêmement fertile. Force est de constater que Therrien prêche par l'exemple puisque Jonathan Lessard et lui sont allés à la rencontre de deux développeurs québécois de la première heure. Dans l'article qui ouvre ce numéro, les portraits qu'ils font de deux autodidactes ayant conçu des jeux dans les années 90, Christian Boutin et I-Grec (pseudonyme), ${ }^{4}$ révèlent les bénéfices indéniables de tels entretiens. Autant on en apprend sur l'avènement et la présence des jeux vidéo ainsi que des ordinateurs dans deux petites villes du Québec, autant on comprend les facteurs qui ont poussé des adolescents à se mettre à la programmation alors que l'informatique était loin d'être aussi présente qu'aujourd'hui dans la vie de tous les jours. Entre un jeu de rôle ayant la série Ultima comme modèle, Walls of Bratock (Oracle Software, 1994), et un jeu d'aventure pointez-et-cliquez à la langue vernaculaire — il a d'ailleurs pour titre le juron québécois Tabarnak (I-Grec, 1996) - Lessard et Therrien marquent bien que la question de la québécité repose déjà à l'époque sur les visées de concepteurs oscillant entre leur culture locale 
et une mondialisation des marchés et des communautés, et qu'en somme les postures et les stratégies de ces derniers n'étaient pas très différentes de celles du mouvement indie contemporain.

C'est un portrait de l'industrie du jeu vidéo au Québec que proposent quant à eux Dominic Arsenault et Louis-Martin Guay. Après le Canada dans le chapitre mentionné plus haut, ils se sont concentrés sur la Belle Province. Ils mettent également en relief le fait que des entrevues avec des acteurs du milieu et un vrai travail d'enquête seraient nécessaires pour corroborer les faits qu'ils portent à notre attention et pour interroger les sources disponibles (se résumant pour l'essentiel à quelques sites personnels de premiers créateurs toujours en ligne). Même s'ils ne visent pas une chronologie exhaustive (qui demanderait qu'on y consacre tout un ouvrage), les trois périodes qu'ils distinguent répondent à une démarche historique s'efforçant de penser et d'expliquer les transformations dans le temps de la production vidéoludique québécoise. Cela est saillant dans le passage de la première période, laquelle montre on ne peut mieux « la pluralité et l'éclectisme de l'écosystème » initial, à la deuxième qui est marquée par l'allocation bien connue de crédits d'impôt à Ubisoft. Quant à la troisième période, elle aussi traversée par des changements politiques, elle se caractérise — sous l'impulsion de quatre ordres de faits — par l'élan d'une diversification de l'industrie, avec la naissance en 2016 de la Guilde des développeurs de jeux vidéo indépendants du Québec (maintenant la Guilde du jeu vidéo du Québec) qui devient en «quelques années [...] le plus gros regroupement de ce type au monde, avec plus de 2000 travailleurs dans 200 studios regroupés sous la forme juridique d'une coopérative ». Pour définir ce qu'est aujourd'hui le jeu vidéo au Québec, Arsenault et Guay concluent leur parcours par l'image d'une courtepointe, création artisanale qui possède une longue tradition au Québec. C'est une analogie forte, parce qu'elle renvoie à un travail qui repose encore sur le savoir-faire des artisan.e.s qui la confectionnent et signale l'importance de tisser des liens serrés entre une diversité de composant.e.s.

L'article de Maude Bonenfant et Jonathan Bonneau vient parfaitement illustrer les relations qui se nouent au Québec grâce à un contexte exceptionnel de "partenariats entre les entreprises vidéoludiques et les laboratoires universitaires, les unes et les autres ayant développé une expertise propre ». Rappelant qu'il n'est pas nouveau que les développeurs entretiennent des rapports avec leurs communautés de joueur.se.s et étudient leurs activités, les auteurs mettent l'accent sur l'analyse de données massives comportementales. ${ }^{5}$ Ils brossent à grands traits un tableau des recherches conduites par les entreprises québécoises - triple A et indépendantes pour en marquer des limites, mais surtout pour montrer ce que l'expertise et les méthodes développées dans un laboratoire comme le Laboratoire de recherche en médias socionumériques et ludification de l'Université du Québec à Montréal, lié au groupe de recherche Homo Ludens sur la communication et le jeu, peuvent apporter à la connaissance et à l'examen des pratiques vidéoludiques. Se tournant vers l'avenir, Bonenfant et Bonneau signalent l'importance que prendra l'intelligence artificielle, et notamment les techniques de recherche et d'analyse à l'aide d'algorithmes, dans ce type d'études sur les communautés des joueur.se.s. L'écosystème québécois de l'intelligence artificielle étant également reconnu mondialement, un autre motif de courtepointe se dessine ici.

Regardant non moins vers les temps à venir, la contribution de Laureline Chiapello atteste de la richesse des recherches universitaires et des réflexions théoriques. En reconsidérant les intérêts 
politiques et économiques favorisant l'avènement et la consolidation du secteur vidéoludique québécois, Chiapello vient en quelque sorte poursuivre la réflexion d'Arsenault et Guay. L'auteure nuance le discours des acteurs du milieu qui valorise sans ambages l'innovation de l'industrie québécoise du jeu vidéo. En abordant la notion de créativité à partir d'un modèle socioculturel constructiviste qui différencie quatre dimensions de cette capacité d'imaginer et d'inventer (à savoir le produit, la personne, le processus et l'environnement), elle en expose les tenants pour mieux en préciser les aboutissants. Notamment, si le directeur créatif est souvent présenté comme "un créateur tout-puissant», on constate en fin de compte que «la compréhension de ce que font les designers reste [...] floue pour les designers eux-mêmes ». En utilisant comme premier exemple le jeu en ligne The Secret World (Funcom, 2012), dont l'échec commercial a entre autres été attribué par son concepteur de jeu en chef à sa trop grande innovation, Chiapello montre la complexité des nœuds qu'elle essaie de dénouer.

En réfléchissant à l'importance des ventes dans la définition d'un jeu créatif, et à la difficulté pour les propriétés intellectuelles des petits studios indépendants de répondre à ce critère, Chiapello souligne : «On peut tout de même citer des jeux indépendants développés à Montréal qui se sont très bien vendus: Fez (Polytron Corporation, 2012) avec plus de un million d'exemplaires, ou le premier jeu de la franchise Outlast (Red Barrels, 2013) avec quatre millions de copies écoulées». C'est précisément aux jeux de Red Barrels, studio qui «démontre le dynamisme de la création indépendante québécoise et sa vivacité au-delà de l'hégémonie exercée par les grandes multinationales », que Jean-Charles Ray consacre son article. L'auteur trempe sa plume dans la peur afin de traduire l'effrayante expérience que vont vivre les joueur.euse.s forcé.e.s. d'explorer l'hôpital psychiatrique du premier opus et de son extension et le village isolé dans le second opus. En concentrant son analyse concise, mais érudite, sur la thématique du regard, il traie d'un récent sous-genre en jeu vidéo, le jeu de fuite à la première personne, au cœur du « récent renouveau de l'horreur vidéoludique ».

Lorsqu'Adam Lefloïc Lebel, que j'ai cité en introduction, soutient que c'est à l'industrie qu'on pense lorsqu'on discute du jeu vidéo au Québec, c'est pour affirmer que celle-ci reste dépendante des joueurs qui la soutiennent. Lefloïc Lebel s'intéresse en particulier à une pratique vidéoludique assidue et à une communauté très affairée qui sont pourtant souvent oubliées, à savoir l'activité, ou la passion devrait-on dire, de collectionner des jeux vidéo. Voyant d'abord à circonscrire ce qu'est le collectionnement, il s'attache ensuite à caractériser celui ou celle qui par goût rassemble des jeux vidéo pour leur qualité esthétique, leur intérêt, leur valeur réelle ou sentimentale ou leur rareté. Étant lui-même ce qu'il nomme un ludovidéophile et souhaitant mieux comprendre sa communauté francophone, Lefloïc Lebel a réalisé une enquête auprès de membres de trois groupes ou clubs de la Belle Province. Il analyse les réponses à son questionnaire en tenant compte des rapports économiques, culturels et sociaux qui sous-tendent la conduite de ludovidéophiles québécois.e.s s'activant dans un marché plutôt mondial, dominé par la vente et l'achat sur Internet. Sans connaître l'analogie utilisée par Arsenault et Guay, l'auteur met la dernière main à son texte en attestant qu'à tout prendre, « la communauté [reste] tricotée serré ».

Dans la mesure où on ne peut « étudier une culture en la détachant de la communauté où elle émerge », et comme un phénomène devient plus ou moins présent dans une culture en fonction de la valeur qu'on lui donne, Francis Lavigne vient éclairer de quelle manière la présence du jeu 
vidéo a pris forme et s'est structurée au Québec. Il concentre sa recherche sur le catalogue général de Bibliothèque et Archives nationales du Québec (BAnQ) puisque cette institution acquiert, rassemble, conserve et diffuse le patrimoine documentaire québécois. Ce sont donc les discours publics en français - la question de la langue n'est jamais évacuée au Québec - qui intéressent l'auteur, tout comme leur accessibilité et leur préservation. Lavigne propose aussi une périodisation en trois temps, mais différente de celle d'Arsenault et Guay. Le passage de la publication papier aux documents sur supports virtuels, l'arrivée du Vidéoway de Vidéotron, un « décodeur » ou système de divertissement télévisuel câblodistribué et interactif qui a marqué l'imaginaire d'une génération, la publication d'un petit nombre de magazines québécois de jeu vidéo et les incontournables préoccupations économiques de l'industrie comptent parmi les faits qui jalonnent l'avènement de la culture vidéoludique québécoise.

\section{Un intérêt certain}

En préparant ce numéro sur le jeu vidéo québécois, j'ai voulu apporter ma modeste contribution à la réflexion. Car si, peu importe la nationalité, le jeu vidéo est aujourd'hui considéré comme un phénomène culturel de masse important, tout autant que la télévision et le cinéma, et que les sciences du jeu se sont constituées en un champ d'études reconnu par les universités, ce ne fut pas toujours le cas. Leur légitimation a indiscutablement été graduelle. Conséquemment, en complément au travail de Francis Lavigne, je me suis tourné vers le discours savant québécois pour y chercher des textes universitaires précurseurs. J'ai recouru au moteur de recherche des universités de la Belle Province et consulté les entrées bibliographiques des textes trouvés, où j'ai croisé des auteur.e.s de la première heure. Comme mes collègues, je suis loin de prétendre épuiser le sujet, marquant plutôt les balises d'une strate de base. Ayant remonté en 1982, je me suis focalisé sur la première décennie et vais terminer ma course en 1991.

Dans le premier texte que j'ai pu repérer, «Contribution à une critique multidimensionnelle du jeu électronique » (1982), Yvan Leroux - alors étudiant au doctorat à l'École de psychologie de l'Université Laval - emploie un néologisme utilisé l'année précédente dans le magazine Jeux et stratégie (Guigo, 1981, p. 19) pour cadrer sa revue de la littérature : il parle de « la ludotique, c'est-à-dire de l'application de l'informatique aux jeux» (1982, p. 87). L'expression «jeu vidéo » n'est évidemment pas encore cristallisée. ${ }^{6}$ C'est davantage vers les jeux électroniques ou la micro-informatique ludique (Leroux et Pépin, 1986, p. 7) qu'on va pointer. Leroux en fournit indirectement une raison :

Le jeu électronique, tel un caméléon techno-social, adopte diverses formes de maquillage : il y a le jouet portatif à piles; le jeu vidéo comprenant une console que l'on branche sur son téléviseur et alimentée par des cassettes de jeu vidéo préprogrammées; le jeu électronique qui se déroule sur micro-ordinateur; le jeu vidéo qui se joue debout dans certains bars, centres commerciaux ou dans les salles d'amusement public ; offert en option sur certaines montres ou calculatrices électroniques ; le jeu pseudo-communautaire - un joueur actif et une multitude de spectateurs, pourtant anonymes les uns pour les autres - rendu possible par le couplage du téléphone à clavier avec un canal de télévision ; etc.

Yvan Leroux, p. 90 (1982) 
En 1983, dans son article «Les jeux électroniques et les salles de jeux : essai d'analyse », Jean Archambault observe à son tour : "L'électronique a aussi envahi le domaine du jeu : le jouet électronique, le jeu vidéo et le jeu électronique en salle publique en sont les représentants » (p. 81). L'objet d'étude est vu comme hétéroclite, mariant l'informatique, l'audiovisuel et l'amusement. Il n'est pas encore pensé en termes d'industrie, mais comme un divertissement. Le jeu d'arcade n'est toutefois pas nommé. En ce sens, Pierre Sormany parlait du lieu, les arcades, et des jeux vidéo (1982, p. 6). Le terme est en fait proscrit par Sûreté : le magazine de la Sûreté du Québec auquel fait référence Archambault (sans citer le passage). On y lit :

Un terme à éviter : « arcade »

C'est sous l'influence de l'anglais que ce mot s'est infiltré dans notre langue. Or, de l'avis de plusieurs terminologues consultés, l'expression à privilégier serait celle de «salle de jeux automatiques » ${ }^{7}$, plutôt que «centre d'amusement» ou « salle d'amusement », locutions rejetées parce qu'elles constituent des calques de l'anglais.

Sans auteur, « Dossier : les salles de jeux automatiques », p. 11 (1983)

Cependant, ladite proscription ne sera pas retenue. Arsenault et Guay marquaient dans ce numéro l'importance du terme " arcade » pour caractériser Têtards. Et dans son mémoire de maîtrise en psychologie à l'Université de Montréal, l'une des premières recherches aux cycles supérieurs sur ce thème, Valérie Clift sépare son échantillon de sujets «en deux groupes expérimentaux, joueurs d'arcade et joueurs maison » (1985, p. vii). Cette fois à l'Université du Québec à Montréal, un autre mémoire de maîtrise en psychologie propose une Étude descriptive des arcades de jeux vidéo. Analyses proxémiques, cognitives et de contenu (1987). Son auteure, Josette Giroux, va de même étudier en anglais les « Arcade Video Games » (1989), et ce, avec Claude M. J. Braun, un collègue de l’Université Concordia.

Les chercheur.e.s se penchent sur un objet contesté qui n'a pas encore été vraiment abordé. Après avoir survolé les écrits à sa disposition en faisant le bilan des pour et des contre, Leroux considère «le besoin d'introduire au sein du débat plus de rigueur critique » $(1982$, p. 89). Ce sera son premier objectif : "En un premier temps, on a dénoncé le peu d'intérêt réel manifesté par les scientifiques à l'égard de l'émergence du jeu électronique. Ce constat étant lié à la récence du phénomène, il est permis de croire que l'avenir devrait s'enrichir d'apports nouveaux et substantiels en ce domaine » (p. 104). Clift fait le même bilan :

En conclusion, il est plutôt difficile de faire le point sur la controverse soulevée par les jeux vidéo. D’une part, derrière les éloges se cachent des intérêts économiques, d'autre part, la littérature scientifique sur le phénomène n'abonde pas. Le phénomène des jeux vidéo n'en perd pas moins de son intérêt et il importe de tenter de saisir les dimensions psychologiques sous-jacentes à l'engouement de millions d'individus à travers le monde pour ce type de jeux.

Valérie Clift, p. 24 (1985)

Braun $^{8}$, Goupil, Giroux et Chagnon traitent, dans «Adolescents and Microcomputers: Sex Differences, Proxemics, Task and Stimulus Variables », de la littératie informatique (computer literacy) en général. Ils notent qu'il ne fallait pas qu'elle se développe seulement à la maison où 
l'ordinateur devenait plus présent (c'est durant cette période que Christian Boutin et I-Grec signalent à Lessard et Therrien leur rencontre avec un TRS-80 et un Tandy 1000), mais qu'elle devrait s'accroître quand plus d'ordinateurs seraient installés dans les écoles. Le jeu vidéo s'intégrant à cette révolution informatique, les chercheur.e.s constatent : «Video game use does not correlate significantly enough with academic performance or social class to warrant alarm. Much consciousness raising is required for communities to cut down on sex bias, violence, and social withdrawal associated with video games and the related literature» (1986, p. 541). Quand elle rédige son mémoire de maîtrise l'année suivante et qu'elle se donne comme but de « mettre en évidence les caractéristiques des jeux vidéo qui pourraient rendre intéressants et captivants les logiciels académiques en milieu scolaire » (1987, p. ii), Giroux n'utilise que des références en anglais ${ }^{9}$ et sa bibliographie ne contient que 27 entrées, en très grande majorité des articles. Cela ne l'empêche pas d'affirmer : "Bien qu'à l'heure actuelle peu de recherches aient porté sur les jeux vidéo, il n'en demeure pas moins qu'en raison de leur grande popularité, ces jeux représentent un intérêt certain » $(1987$, p. 2). On prend conscience que le phénomène n'est pas un éphémère et tisse par le fait même les premiers morceaux des études du jeu vidéo.

Sormany ouvre son article « Les machines s'amusent-elles? », dans Les Diplômés: La Revue des Diplômés de l'Université de Montréal, de cette façon :

$\mathrm{Au}$ début, ce n'était qu'un petit trait blanc, la palette, et un petit point mobile, la balle, qu'il fallait intercepter. Un divertissement anodin, qui sévissait dans les salles d'attente des gares, en attendant d'envahir le petit écran domestique. « Mais on s'en lasse si vite ", disaient les prophètes, après s'être laissé séduire par le ping-pong électronique.

Huit ans plus tard, palettes et balles ont pris la forme de fusées interplanétaires, de monstres intergalactiques ou d'animaux sympathiques, voyageant dans des univers à trois dimensions où les obstacles défilent sur vous, en relief et en couleurs, dans des scénarios constamment renouvelés.

Pierre Sormany, p. 6 (1982)

On ne se détournera donc pas des écrans jouables. Dans « Les jeux électroniques et les salles de jeux : essai d'analyse », et pour preuve que la recherche sur le sujet n'était pas foisonnante, Archambault se réfère à une constatation de Claude Boies dans Sûreté : le magazine de la Sûreté $d u$ Québec $^{10}$ pour mettre l'accent sur l'incontournable place qu'ont prise les activités vidéoludiques dans les arcades.

Il faut se rendre à l'évidence : les jeux vidéo sont là pour rester. Il s'agit de définir, en tant que société, comment nous voulons les utiliser. Il est important que chaque parent prenne conscience de l'existence du phénomène de même que de toutes les implications et les conséquences qui s'y rattachent.

Claude Boies, p. 19 (1983) 
Brazeau, Ménard-Burrows et Lussier-Laurenzi déplacent leurs observations dans un autre lieu, mais arrivent à la même conclusion : "Tout indique que le progrès technique va se poursuivre. Les jeux électroniques vont bientôt se répandre partout et ils deviendront de plus en plus sophistiqués. L'école se doit de les apprivoiser, de les analyser et d'en découvrir les applications pédagogiques » $(1985$, p. 22). Sans conteste, les auteures étaient de meilleures prophétesses que les prophètes raillés par Sormany.

Alors que l'âge moyen du joueur canadien en 2020 est de 34 ans et que $50 \%$ des « joueurs » au pays sont des femmes et $50 \%$ des hommes (Association canadienne du logiciel de divertissement, 2020, p. 7), on aura deviné que ce n'était pas le cas dans les années 1980. C'est ce qui explique que les textes qui forment mon petit corpus soient des études en psychologie et en psychoéducation. On se penche sur les effets du jeu vidéo en général sur les enfants et les élèves. Pour parler de ces enfants de la génération de l'ordinateur, Sormany utilise le terme introduit dans le magazine Time: les microkids (182, p. 7). Dès lors que les parents ne s'adonnent pas encore énormément aux activités vidéoludiques, Jacques de Lorimier décèle une « guilde d'initiés » (1991, p. 57). Il admet avoir voulu écrire Ils jouent au Nintendo... Mais apprennent-ils quelque chose? en regardant jouer son fils et ses amis. Il termine son ouvrage par un encouragement : "Autant se préparer aujourd'hui, avec des jeux encore relativement peu performants, à accompagner les enfants et les adolescents dans un périple qu'ils seront seuls à pouvoir accomplir » (p. 154, italiques de 1'auteur).

À l'inverse, de Lorimier amorce son essai en pédagogie familiale en affirmant :

Ce livre aborde un phénomène récent : l'introduction des jeux vidéo dans les foyers. Un tel phénomène devient envahissant; aussi mérite-t-il qu'on s'y arrête. Les parents et les éducateurs des fans de jeux vidéo domestiques trouveront, dans les pages qui suivent, des observations et des réflexions sur ce que d'aucuns appellent une maladie : la «nintendinite» ou la passion du Nintendo.

Jacques de Lorimier, p. 9 (1991)

Chaque époque et chaque nouveau média ont connu leur part de panique morale. Ce ne fut pas différent pour le jeu vidéo dans les années 1980. Ceux qui jouent sont considérés comme « les nouveaux drogués de l'électron». Et Sormany de poursuivre alors qu'il signale la percée des « ordinateurs de jeu vidéo (Atari, Intellivision, etc.)» et des micro-ordinateurs domestiques : «En attendant cette invasion ultime du loisir cathodique, le monde se divise en deux groupes: ceux qui refusent les jeux vidéo, qui ne comprennent pas l'engouement des jeunes, qui dénoncent... et ceux qui ont été piqués » (1982, p. 6, italiques de l'auteur). Giroux et Leroux vont dans cette optique affirmer que les « individus deviennent intoxiqués » (Giroux, 1987, p. 15) et que c'est une intoxication psychologique par la «caféine électronique », et non pas par de la « cocaïne électronique » (Leroux, 1982, p. 94). ${ }^{11}$ Surtout, comme le fait remarquer Sormany, « dans les milieux policiers, les arcades ont bien mauvaise réputation » $(1982$, p. 6). À n'en pas douter, le magazine de la Sureté du Québec est tranchant. Le titre de l'article de Claude Boies pose l'analogie qui marque les esprits : «Casinos pour jeunes?» L'avocat cite une étude psychologique selon laquelle les jeux vidéo empoisonnent les enfants, et il met l'accent sur l'intérêt grandissant de ces loisirs en salles de jeux automatiques. Or, il se demande : «Comment 
accepter sans réaction que des jeunes fréquentent des salles où on y retrouve, dans certains cas, un réseau de drogues fort bien structuré; ou encore, où d'autres jeunes se rassemblent pour préparer le prochain 'coup' qu'ils exécuteront; ou bien l'endroit de prédilection de receleurs » (1983, p. 21). Dans cette perspective, aller jouer « innocemment» à Pac-Man (Namco, 1980) peut mener au crime: on commence par voler ses parents pour assumer les coûts de cette pratique et on finit en vendant ses charmes à des adultes. ${ }^{12}$ Et Boies d'observer : " On bloque des projets gouvernementaux visant à établir des casinos de peur que le taux de criminalité n'augmente (les études le prouvent), mais on laisse « pousser », à un rythme effarant, ces salles qui ne sont ni plus ni moins que des casinos pour jeunes adolescents » (1983, p. 22). Certes les bornes d'arcade ressemblaient par leur forme à des machines à sous. Le capitaine Roland Labisssonnière, chef du Bureau de l'alcool, de la moralité, des drogues et du jeu à Montréal, utilise aussi cette analogie dans « Polyvalente du crime » :

En résumé, la forte proportion des salles de jeux automatiques est devenue néfaste pour notre jeunesse. Pendant qu'on prend beaucoup de précautions pour intégrer les «gros» casinos pour adultes, nous permettons, par notre inertie, à nos enfants d'avoir le plus beau réseau de maisons de jeux exploitées de la façon la plus arbitraire qui soit, en plus de permettre qu'on fasse de l'argent sur leur dos.

Roland Labissonnière, p. 12 (1983)

Si on ne se lassera pas des jeux vidéo, de telles réactions s'opposant vivement aux pratiques vidéoludiques ne cesseront pas non plus. Difficile de ne pas faire un parallèle ici entre cette idée de casino et les coffres à butin, ou loot boxes. C'est au Québec, en octobre 2019, que des parents ont déposé une demande d'action collective contre Epic Games et son Fortnite, alléguant que les créateurs avaient recours "aux mêmes tactiques que les créateurs de machines à sous » pour concevoir des mécaniques destinées à harponner les joueur.euse.s (Lecomte, 2019 ; Johnson et Ivany, 2021). Offrant des récompenses aléatoires, les boîtes virtuelles sont liées à la dépendance aux jeux de hasard (BBC, 2021).

Comme l'annonçait Leroux, les chercheur.e.s abordent déjà le jeu vidéo — « trop vite lapidé? » (Leroux, 1982, p. 89) — avec une rigueur critique tout en voulant éviter les généralisations pour mieux le définir. Sormany nuance : « On peut dénoncer ce loisir solitaire, anti-social même. On peut aussi y voir quelque vertu, comme ce physicien qui dans New Scientist, au printemps dernier, affirmait que les enfants de la génération vidéo pouvaient mieux comprendre la réalité de la physique quantique » (1982, p. 6-7). Archambault affirme également : « Sans nier que les salles de jeux puissent favoriser certains comportements criminels, nous sommes d'avis qu'une analyse expérimentale de la situation permettrait de comprendre, d'agir sur elle davantage et de mieux la contrôler, ce qui nous éviterait peut-être de jeter le bébé avec l'eau du bain! » (1983, p. 85). Leroux relève quant à lui les répercussions paradoxales du phénomène. " Le jeu électronique est januséen : d'une part, il est un thermomètre indiquant la surchauffe sociale, un malaise dans la civilisation et agit comme médiateur de l'acculturation; d'autre part, il peut être considéré à certains égards comme un exutoire salutaire pour l'individu baignant dans cette société » (1982, p. 91). Dans « Jeu sur micro-ordinateur et différences liées au sexe », Leroux et Pépin marquent d'un trait la disparité de la pratique entre les garçons et les filles; citant des 
collègues américains, ils signalent de manière sagace : "c'est une erreur de considérer l'ordinateur comme un agent neutre de socialisation » (1986, p. 180).

En citant un article de l'année précédente, ${ }^{13}$ Leroux pointe vers un aspect révolutionnaire du jeu vidéo : «Le jouet électronique n'est plus un simple objet-jouet, il devient un objet-joueur » (1982, p. 96). En rapportant les propos de Louis-Philippe Hébert, de Logidisque, Somary voit le jeu vidéo comme une sorte de littérature, car " on peut placer là-dedans les mêmes ressources imaginaires que dans un roman conventionnel » (1982, p. 7). Pour de Lorimier, le Nintendo reste un «jeu interactif» domestique qui est «un médium polyvalent» avec des images qui deviennent réalité. Devant Blades of Steel (Konami, 1987), il s’extasie :

On se croirait sur l'une ou l'autre des patinoires de la Ligue nationale où l'on est en train de filmer une joute en télé-vidéo. Les joueurs tournoient autour de la rondelle, les arbitres sifflent et procèdent à la mise au jeu après un point compté, une punition ou un hors-jeu, la foule crie, on entend le crissement des patins sur la glace. C'est aussi vrai que vrai.

Jacques de Lorimier, p. 18 (1991)

Les « cassettes » du Nintendo font appel à un imaginaire qui n'est plus comme celui des adultes. Ainsi, l'imaginaire des jeunes est actif et formé par des images en mouvement qui alimentent une esthétique particulière apparentée à la réalité ; il est participatif et friand de variété. Somme toute, «le titre de ce livre aurait d'ailleurs pu être Le Nintendo ou l'imaginaire sans les mots" (1991, p. 105). Enfin, pour le spécialiste en sciences de l'éducation, il faut prendre conscience que notre imaginaire reste domestiqué, par l'école quand on est enfant, et par le travail une fois à l'âge adulte. C'est ce qui explique en partie l'attrait du jeu vidéo:

Face à cet imaginaire domestiqué, les jeunes qui jouent au Nintendo s'accordent un répit de quelques années avant d'être absorbés, à leur tour, dans l'univers des adultes. [...] Mais, quel qu'il soit, cet imaginaire sollicite les adultes à relever un double défi: entrer, comme éducateur, dans l'imaginaire des jeunes et tenter de le comprendre; cultiver notre propre imaginaire en le libérant de la domestication.

Jacques de Lorimier, p. 128-129 (1991)

Cette recommandation de cultiver son imaginaire, de le diversifier, résonne encore aujourd'hui.

On réalise en consultant les écrits des années 1980 à quel point il a fallu du temps pour que le jeu vidéo devienne une affaire sérieuse, quelque chose à étudier. On constate également que ce qui intéresse surtout, ce sont les pratiques vidéoludiques et leurs effets chez les jeunes joueurs. Les objets ne sont pas au départ le centre d'intérêt; il n'y a en effet pratiquement pas de jeux nommés dans les articles et encore moins d'analyses formelles. ${ }^{14}$ Il faudrait poursuivre l'investigation pour exposer en détail l'évolution du discours universitaire québécois sur le jeu vidéo.

Il est fort révélateur que les auteurs et autrices de quatre articles de ce numéro - cinq si l'on inclut ma courte contribution - disent ne pas prétendre à l'exhaustivité, aspirant plutôt à poser des jalons pour la recherche et à donner des exemples de ce qui se fait et de ce qui est à faire. L'ensemble du numéro souhaite de la sorte encourager l'examen critique et l'histoire locale du 
jeu vidéo québécois encore embryonnaire. Cela est nécessaire et fondamental, surtout au pays du Je me souviens.

\section{Références}

Aarseth, E. (2001). Computer Game Studies, Year One. In Gamestudies, 1(1), Juillet. En ligne: http://www.gamestudies.org/0101/editorial.html.

Archambault, J. (1983). Les jeux électroniques et les salles de jeux: essai d'analyse. In Revue de modification du comportement, 13(2), été, 81-87.

Arsenault, D. et Guay, L.M. (2015). Canada. In Mark J.P. WOLF (dir.), Video Games Around the World, (p. 105-118). Cambridge (MA): MIT Press.

Asselin, P. (2009). Francisation réussie du jeu vidéo. In Le Soleil numérique, 15 septembre. En ligne: https://www.lesoleil.com/affaires/techno/francisation-reussie-du-jeu-video4b288a06deb20c2e9fa8041d8df51c2a.

Association canadienne du logiciel de divertissement (ADL) (2020). Amateurs de jeux vidéo au Canada Faits essentiels 2020. En ligne: https://essentialfacts2020.ca/wpcontent/uploads/2020/11/RCGEF_fr.pdf.

BBC (2021). Jeux vidéo : les coffres à butin liés à la dépendance aux jeux de hasard, selon une étude. Radio-canada.ca, 2 avril. En ligne : https://ici.radiocanada.ca/nouvelle/1781994/jeux-video-loot-boxes-coffres-butin-dependance-etudee.

Blanchet, A. et G. Montagnon (2020). Une histoire du jeu vidéo en France. 1960-1991 : Des labos aux chambres d'ados. Éditions Pix'n Love, Houdan.

Boies, C. (1983). Casinos pour jeunes? In Sûreté : le magazine de la Sûreté du Québec, 13(2), février, 19-22.

Braun, J., G. Goupil, J. Giroux et Y. Chagnon (1986). Adolescents and Microcomputers: Sex Differences, Proxemics, Task and Stimulus Variables. In The Journal of Psychology, 120 (6), 529-542.

Braun, C.M.J. et J. Giroux (1989). Arcade Video Games: Proxemic, Cognitive and Content Analyses. In Journal of Leisure Research, 21(2), 92-105.

Brazeau, L., C. Ménard-Burrows et F. Lussier-Laurenzi (1985). Jeux sur micro-ordinateur. In Québec Français (L’Acadie : littérature et culture), 60, décembre, 22-24.

Clift, V. (1985). La recherche de sensations et de pouvoir à travers la pratique des jeux vidéo, mémoire de maîtrise, département de psychologie, Université de Montréal, septembre.

De Lorimier, J. (1991). Ils jouent au Nintendo... Mais apprennent-ils quelque chose? Éditions Logiques, Montréal. 
Della Rocca, J. (2013). The Montreal Indie Game Development Scene...Before Ubisoft. In Loading... Journal of the Canadian Game Studies Association, 7(11), Special Issue: 'Indie, Eh?', 130-132.

Donovan, T. (2010). Replay. The History of Video Games. East Sussex: Yellow Ant.

« Dossier : les salles de jeux automatiques ». (1983). In Sûreté : le magazine de la Sûreté du Québec, février, 11-22.

Grandadam, D., P. Cohendet, et L. Simon (2012). Places, Spaces and the Dynamics of Creativity: The Video Game Industry in Montreal. In Regional Studies, 47(10), 1701-1714. doi:10.1080/00343404.2012.699191.

Giroux, J. (1987). Étude descriptive des arcades de jeux vidéo. Analyses proxémiques, cognitives et de contenu, mémoire de maîtrise, département de psychologie, Université du Québec à Montréal, novembre.

Guigo, D. (1981). Quel avenir pour les jeux sur ordinateur? In Jeux et stratégie, 11, 19-20, et 2226.

Herman, L. ([1994] 2009). Phoenix: The Fall \& Rise of Video Games, 3e édition. Springfiled: Rolenta Press.

Johnson et Ivany, (2021). Un document interne d'EA révélerait une stratégie pour inciter les joueurs à dépenser. In Radio-canada.ca, 26 avril. En ligne: https://ici.radiocanada.ca/nouvelle/1787686/electronic-arts-lootbox-jeu-video-coffres-butin-hasard.

Kent, S. L. (2001). The Ultimate History of Video Games. New York: Three Rivers Press.

Labissonnière, R. (1983). Polyvalentes du crime. In Sûreté : le magazine de la Sûreté du Québec, 13(2), février, 12-14.

Lecomte, A.M. (2019). Des avocats montréalais partent en guerre contre le jeu Fortnite. Radiocanada.ca, 19 octobre. En ligne: https://ici.radio-canada.ca/nouvelle/1331640/fortnitejeu-video-action-collective.

Leroux, Y. (1982). Contribution à une critique multidimensionnelle du jeu électronique. In Revue canadienne de psycho-éducation, 11(2), 87-109.

Leroux, Y. et M. Pépin (1986). Jeu sur micro-ordinateur et différences liées au sexe. In Revue des sciences de l'éducation, 12(2), 173-196.

Lessard, J. (2013). Glutomax: Quebecois Proto-Indie Game Development. In Loading... Journal of the Canadian Game Studies Association, 7(11), Special Issue: 'Indie, Eh?', 133-138.

Manenti, B. (2011). Montréal, la Mecque du jeu vidéo. In Le Nouvel Observateur. 24 août. En ligne: http://teleobs.nouvelobs.com/jeux-video/20110824.OBS9056/montreal-la-mecquedu-jeu-video.htm.

Pineault, Y. (2015). Le jeu vidéo à Montréal: une négociation entre création et production. In Sciences $d u$ jeu, 4, 1-17. En ligne : https://journals.openedition.org/sdj/494?lang=en. 
Prémont, C. (2009). Guide de l'industrie jeux vidéo, 1 ${ }^{\mathrm{er}}$ édition. Montréal, Québec: Le Lien Multimédia.

Sormany P. (1982). Les machines s'amusent-elles ? In Les Diplômés : La Revue des Diplômés de l'Université de Montréal,341, décembre, 6-8.

TechnoCompétences (2016). Profil de la main-d'œuvre dans l'industrie du jeu électronique au Québec en 2016. En ligne: https://www.technocompetences.qc.ca/wpcontent/uploads/2018/11/2016_Profil_MO JeuVid\%C3\%A9o_TECHNOComp \%C3\%A9t ences.pdf.

Therrien, C. (2015). Inspecting Video Game Historiography Through Critical Lens: Etymology of the First-Person Shooter Genre. In Game Studies: the international journal of computer game research, 15(2), Décembre. En ligne: http://gamestudies.org/1502/articles/therrien.

William, J.F. (2001). L'industrie québécoise. Almanach du jeu vidéo (p. 243-260). Montréal: Les Éditions Logiques.

Wolf, M. J. P. (2008) (dir.). The Video Game Explosion. A History from PONG to PlayStation and Beyond. Westport: Greenwood Press.

${ }^{1}$ La ville était déjà vue il y a dix ans comme « la Mecque du jeu vidéo » par Le Nouvel Observateur (Manenti, 2011).

${ }^{2}$ Il y a bien un chapitre sur " l'industrie québécoise » dans l'Almanach du jeu vidéo de Jean-François William (2001) et un Guide de l'industrie jeu vidéo (2009), écrits respectivement par un chroniqueur de jeux vidéo au Journal de Montréal à l'époque et un journaliste. On retrouve également des articles sur ladite industrie dans des revues spécialisées comme Les Affaires ou Convergence (seul magazine québécois entièrement consacré à la culture et à l'entreprise numérique et technologique, $<$ http://www.lienmultimedia.com/rubrique28 $>$ ) et dans des rapports gouvernementaux, des profils de marché (entre autres par TechnoCompétences, 2016) ou des faits essentiels signalés par l'Association canadienne du logiciel de divertissement (ADL) (2020). Mais dans la majorité des cas, les auteurs de ces documents ne réfléchissent pas de manière globalisante aux informations et aux faits présentés.

${ }^{3}$ Suivant la clause 52.1 du chapitre C-11 de la Charte de la langue française : « Tout logiciel, y compris tout ludiciel ou système d'exploitation, qu'il soit installé ou non, doit être disponible en français, à moins qu'il n'en existe aucune version française. Les logiciels peuvent être disponibles également dans d'autres langues que le français, pourvu que la version française soit accessible dans des conditions, sous réserve du prix lorsque celui-ci résulte d'un coût de production ou de distribution supérieur, au moins aussi favorables et possède des caractéristiques techniques au moins équivalentes » (Voir http://legisquebec.gouv.qc.ca/fr/showversion/cs/C11? code $=$ se $: 52$ 1\&pointInTime $=20170225 \#$ 20170225). Sans version française, un logiciel peut être commercialisé quand même. Je remercie Dominic Arsenault pour cette précision.

${ }^{4}$ Jonathan Lessard avait au préalable introduit leurs œuvres dans son texte " Glutomax: Quebecois Proto-Indie Game Development » (2013).

${ }^{5} \mathrm{Au}$ moment de rédiger l'article, Maude Bonenfant est titulaire de la Chaire de recherche du Canada sur les communautés de joueurs et les données massives (2018-2023).

${ }^{6}$ L'expression l'est certainement plus aujourd'hui, sans toutefois qu'il y ait uniformisation parfaite. Dans le milieu anglophone notamment, tandis qu'Espen Arseth annonce la « Computer Game Studies, Year One » (Aarseth, 2001), l'organisation internationale qui sera fondée en 2003 se nomme la Digital Games Research Association (DiGRA). Et on emploie autant video game que videogame en un seul mot.

7 Cette formulation est peut-être influencée par l'industrie française puisque qu'on parlait du secteur de l'« automatique de divertissement» pour regrouper entre autres les juke-box, les jeux de foire, les flippers 
(ou « machines à boule » en bon québécois) et les jeux électromécaniques. Voir Blanchet Montagnon, 2020, p. 54-55.

${ }^{8}$ Braun est à ce moment affilié à l’Université du Québec à Montréal.

${ }^{9}$ Il y a une exception, un article dans Pour la science reprenant en français les travaux de Lynn A. Cooper et Roger N. Shepard, chercheurs américains en psychologie, sur le retournement mental des objets (février 1985).

${ }^{10}$ C'est par l'intermédiaire d'Archambault que j'ai consulté Sûreté : le magazine de la Sûreté du Québec. Bien que ce ne soit pas une revue universitaire, j'y renvoie tout de même parce que ledit magazine est une source d'un texte savant.

${ }^{11}$ Leroux apporte une précision en note: «Cette notion d'intoxication psychologique se réfère à une forme pathologique relativement mineure comparativement au "gambling", qui est le fief des jeux de hasard et d'argent. Les jeux vidéo sont généralement des jeux d'habileté où l'exercice de l'activité ludique est seule auto-gratifiante. La réalité subjective que nous tentons de décrire est assez bien approchée par la métaphore de " caféine électronique " retrouvée chez Rayl ([Pac-mani!, People Weekly, 1982]) qui illustre l'aspect psychophysiologique «stimulant» du jeu électronique. Ici, on ne parle pas de "cocaïne électronique ". De fait, s'il n'y a pas toxicomanie prononcée (i.e. dépendance psychique et physique à l'égard des effets du jeu), une sur-consommation (relativement aux besoins de satisfaction et à la tolérance de l'organisme) peut favoriser éventuellement l'apparition de troubles de comportement (à un degré de déviance variable) » (p. 107, n. 7).

${ }^{12}$ Ces deux dernières observations sont celles de Labissonnière (1983, p. 14).

${ }^{13}$ C'est l'article de J.M. Peronnet, L'ordinateur contre le jeu?, dans Vers l'éducation de juin-juillet 1981.

${ }^{14}$ Giroux explique avoir observé 21 jeux pour analyser l'hypothèse considérant que les valeurs véhiculées par les jeux vidéo sont anti-sociales (1987, p. 42). Cependant, elle ne détaille pas de quels jeux il s'agit. Ce n'est toujours pas fait dans Brune et Giroux (1989). Clift propose toutefois une courte histoire du jeu vidéo basée sur quelques titres : Space Invaders, Computerspace [sic], Pong, Asteroids, Space Invaders, PacMan [sic], Dragons' Lair [sic pour Dragon's ?] et Match 3 [sic pour M.A.C.H. 3] (1985, p. 11-15). 